\title{
Unmyelinated Nerve Fiber Analysis of the Human Abducent Nerve
}

\author{
By
Nobusuke KOBAYASHI, Yoshiharu SAWABE, Kiyoshi MATSUMOTO, Naruhito OTSUKA* and Noboru GOTO*

\begin{abstract}
Departments of Neurosurgery and Anatomy*, Showa University School of Medicine, 5-8, Hatanodai 1, Shinagawa-ku, Tokyo 142-8555, Japan
\end{abstract}

- Received for Publication, February 12, 1998 -

\begin{abstract}
Key Words: Abducent nerve, Unmyelinated nerve fiber, Morphometry, Aging process, LPH staining method
Summary: Unmyelinated nerve fibers of the abducent nerve have occasionally been observed with electron microscopes, but, to our knowledge, they have never been evaluated from the morphometric point of view. We analyzed the aging process of the unmyelinated nerve fibers in the human abducent nerve with the help of a new staining method suitable for morphometric research on the nervous system. We studied numbers and transverse areas of unmyelinated fibers of the abducent nerve in 10 cadavers. Our findings were that (1) these fibers were distributed diffusely, (2) their number decreased with age, and (3) the mean transverse area did not change with age. Most of the unmyelinated axons were thinner than the myelinated axons. These results may be important for analysis of clinical signs in relation to aging and ophthalmologic functions.
\end{abstract}

Unmyelinated nerve fibers of the abducent nerve have been discovered thanks to the electron microscope $^{6)}$, but no details are known. It is essential that they should be analyzed to improve our understanding of ophthalmologic functions. Therefore, we decided to conduct a study of changes in their numbers and transverse areas in relation with age.

\section{Material and Methods}

The material was obtained from 10 cadavers aged 63-88 years (average age: 74.7 years). The causes of death were not directly or indirectly related to the nervous system including the abducent nerve. Part of the abducent nerve were removed from the subarachnoid space.

The preparation of sections was the same as in our previous reports ${ }^{1-5,7,8)}$ regarding the fixation, washing, dehydration, embedding, sectioning and staining.

\section{Morphometry}

To measure and analyze unmyelinated axons of the abducent nerve, we employed a microscope (BH2, Olympus, Tokyo, Japan), eqipped with a drawing tube (BH2-DA, Olympus, Tokyo, Japan), a digitizer (Crystizer a KC3300, Graphtec, Yokohama, Japan), a computer (PC-9801VX2, NEC, Tokyo, Japan), and an original analysing system program. Highly enlarged images (1,600 times, oil immersion) and a digitizer tablet with a mouse equipped with a point-tip light conducted by an optic fiber were selected to measure the transverse area and perimeter of unmyelinated axons, and to count their numbers. Regression analysis was performed between age and each of the number of unmyelinated fibers, the axonal area and the axonal perimeter.

\section{Results}

\section{Number of unmyelinated fibers}

The total number of unmyelinated fibers in the abducent nerve ranged from 5 to 25 (average 16) within the total area. The data for all cases are listed in Table 1. Unmyelinated nerve fibers were discernible when dark purple (Fig. 1) or that the total number of unmyelinated fibers is reduced with age $(r=-0.90, p<0.001$, Fig. 2$)$. 
Table 1. Unmyelinated fiber analysis of the human abducent nerve

\begin{tabular}{lccccc}
\hline Subject No. & Age & Sex & NUA & MAUA $\left(\mu \mathrm{m}^{2}\right)$ & MPUA $(\mu \mathrm{m})$ \\
\hline 1482 & 63 & F & 25 & 1.39 & 4.95 \\
1463 & 68 & M & 25 & 1.08 & 4.16 \\
1477 & 68 & F & 24 & 1.20 & 4.55 \\
1476 & 71 & F & 16 & 1.04 & 4.55 \\
1483 & 72 & M & 15 & 1.23 & 4.51 \\
1473 & 75 & F & 10 & 1.16 & 4.45 \\
1470 & 77 & F & 18 & 1.26 & 4.73 \\
1466 & 79 & M & 10 & 1.19 & 4.89 \\
1464 & 86 & F & 8 & 1.31 & 4.77 \\
1462 & 88 & M & 5 & 1.22 & 4.60 \\
\hline Mean & 75 & & 16 & 1.21 & 0.23 \\
SD & 8 & & 7 & 0.10 & \\
\hline
\end{tabular}

NUA: number of unmyelinated axons, MAUA: mean area of unmyelinated axons, MPUA: mean perimeter of unmyelinated axons, SD: standard deviation.

\section{Transverse area of unmyelinated axons}

The mean transverse area of unmyelinated axons in the abducent nerve ranged from 1.04 to $1.39 \mu \mathrm{m}^{2}$ (average $1.21 \mu \mathrm{m}^{2}$ ). The regression analysis showed no significant correlation between the axonal transverse area and age $(r=0.093, p>0.10)$.

\section{Perimeter of unmyelinated axons}

The mean perimeter of unmyelinated axons in the abducent nerve ranged from 4.16 to 4.95 (average 4.60) $\mu \mathrm{m}$. No significant correlation between the axonal perimeter and age appears in the regression analysis $(r=0.313, p>0.10)$.

\section{Discussion}

It had been confirmed by electron microscopy that unmyelinated fibers exist in the abducent nerve of the cat and the rat ${ }^{6}$. However, no morphometric research on human unmyelinated fibers have been reported. The reason may be that the various methods previously used for staining the nervous system (myelin sheath stain, luxol fast blue-cresyl violet, etc.) cannot discriminate between axons and other tissue components. Although some silver impregnation methods can distinguish axons, they always result in severe tissue shrinkage. Our new staining method (LPH staining method), is ideal for morphometric research on the nervous system thanks to clear discrimination between axon and myelin sheath and minimized shrinkage (only about $10 \%$ in length ${ }^{33}$ ). We therefore, employed it to examine the morphometric aging process of the unmyelinated fibers in the human abducent nerve.

We found that such fibers were diffusely distributed among myelinated fibers without forming a bundle. We also found that the number of unmyelinated fibers in the abducent nerve is reduced with age. The reason for this, however, remains unknown. Unmyelinated fibers in the abducent nerve may be considered to be autonomic, although their functions have not been elucidated yet. We, however, believe that physiological or histochemical studies can shed some light on their morphology.

The transverse area of unmyelinated axons in the abducent nerve ranged from 1.04 to $1.39 \mu \mathrm{m}^{2}$ without significant changes with age. Experimental research using electron microscopes have shown that the transverse area of unmyelinated axons in abducent nerves ranged from 0.03 to $1.33 \mu \mathrm{m}^{2}$ in the cat and from 0.03 to $2.27 \mu \mathrm{m}^{2}$ in the rat ${ }^{6)}$ (data compiled by us). Our results on the human being showed similar data. Contrary to what other authors have reported ${ }^{1,2,4,5,7,8}$, we found that most of the unmyelinated axons were thinner than myelinated axons. A further area of research in the unmyelinated fibers in the abducent nerve could be to elucidate their function in relation to oculogyric functions, as this still remains unknown to this day.

\section{References}

1) Fujii $M$ and Goto $N$. Nerve fiber analysis of the facial nerve. Ann Otol Rhinol Laryngol 1989; 98:732-735.

2) Fujii $\mathbf{M}$, Goto $\mathbf{N}$ and Kikuchi $\mathbf{K}$. Nerve fiber analysis and the aging process of the vestibulocochlear nerve. Ann Otol Rhinol Laryngol 1990; 99:863-870.

3) Goto N. Discriminative staining methods for the nervous system: Luxol fast-blue periodic acid-Schiff-hematoxylin triple stain and subsidiary staining methods. Stain Technol 1987; 62:305-315.

4) Takeshita $Y$, Matsumoto $K$, Goto $N$ and Shibata $M$. Nerve fiber analysis and aging process of the human oculomotor 
nerve. Showa Univ J Med Sci 1996; 8:55-61.

5) Tang W, Goto $N$, Tanaka $J$ and Otsuka N. Myelinated nerve fibre analysis of the human small splanchnic nerve. Okajimas Folia Anat Jpn 1997; 74:93-98.

6) Tanishima T. Ultrastructural localization of acetylcholinesterase activity inunmyelinated nerve fibers of oculomotor, trochlear and abducens nerve trunks in cat and rat. Report II Studies on the trochlear and abducens nerve trunks.
Japan Ophth 1971; 75:624-634.

7) Yanagisawa $K$, Goto $N$, Kimura $T$ and Tanaka J. Nerve fiber analysis on aging process of deep peroneal nerve in man. J Showa Med Assoc 1994; 54:249-254 (in japanese).

8) Zhang $\mathrm{C}$, Goto $\mathrm{N}$ and Zhou $\mathrm{M}$. Morphometric analysis and aging process of nerve fibers in the human spinal posterior funiculus. Okajimas Folia Anat Jpn 1995; 72:259-264. 


\section{Explanation of Figures}

\section{Plate I}

Fig. 1. High-power view of the human abducent nerve of an 88 year-old man, LPH stain, scale bar $=10 \mu \mathrm{m}$ ( $5 \mu \mathrm{m}$ for the magnified inset) showing unmyelinated fibers. Such fibers are diffusely distributed among myelinated fibers without forming any bundle. 
Plate I

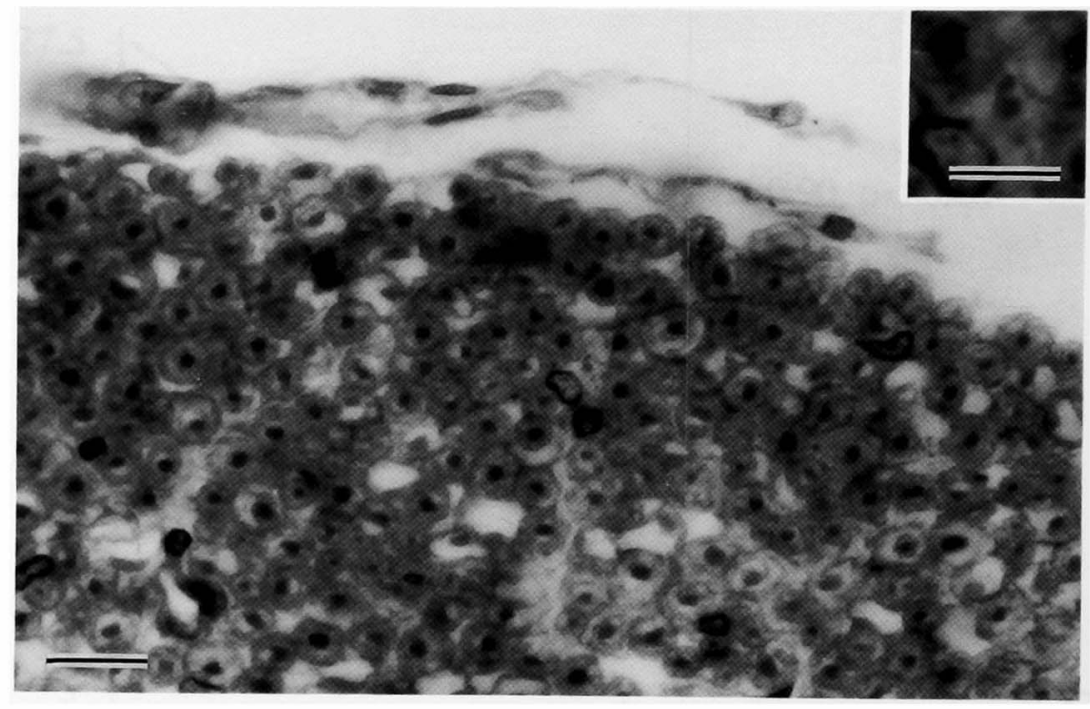


Fig. 2. Scatter diagram and regression line showing a correlation between the number of unmyelinated axons and age in the abducent nerve. 


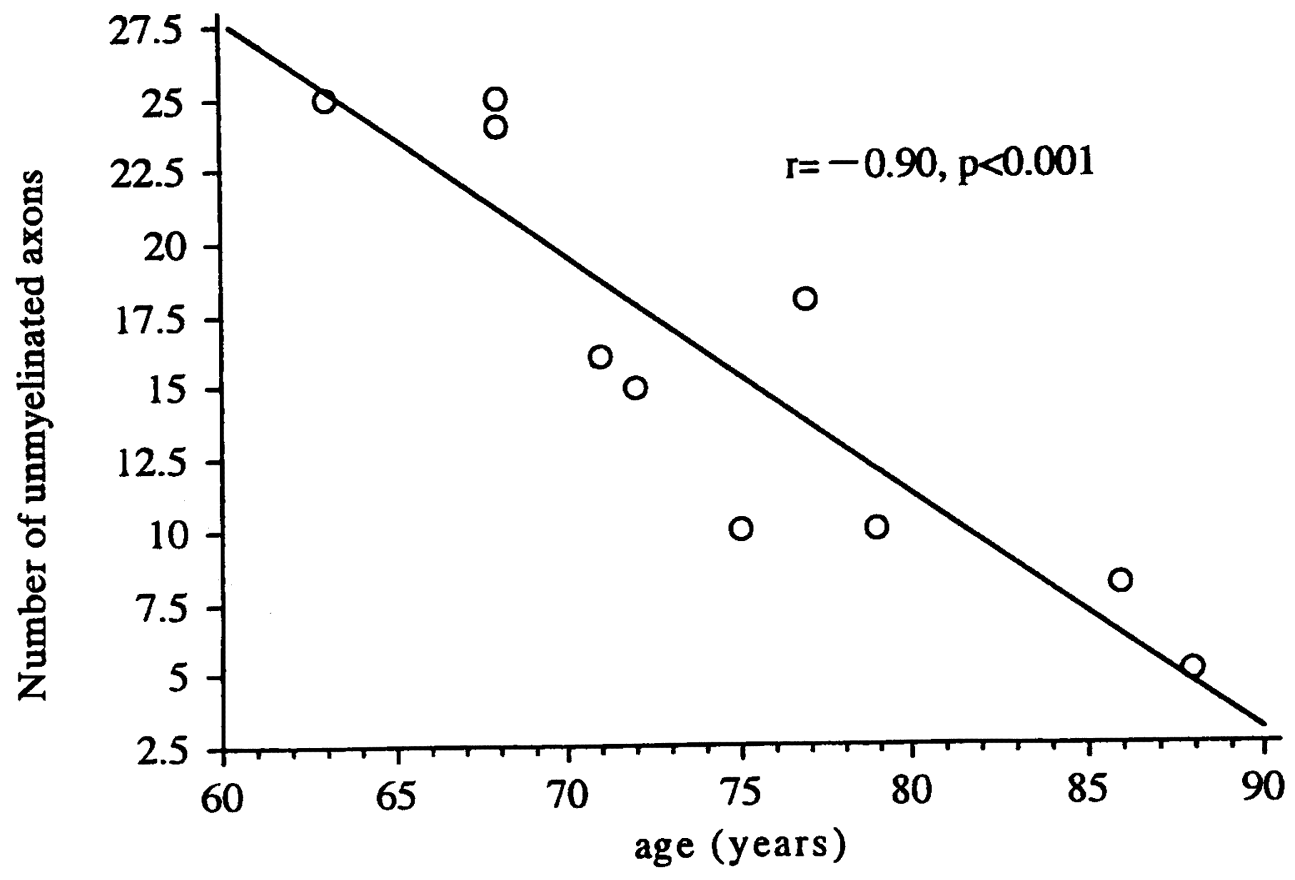

